\title{
Management of Nasal Cavity Chondrosarcoma: About a Case and Review of the Literature
}

\author{
Abboud FZ1* ${ }^{*}$, Bouziane $\mathrm{A}^{1}$, Youssoufi $\mathrm{MA}^{2}$, Alami $\mathrm{Z}^{1}$, Bouhafa $\mathrm{T}^{1}$ and \\ Hassouni $\mathrm{K}^{1}$ \\ ${ }^{1}$ Department of Radiation Oncology, University Hospital Hassan II, Morocco \\ 2Medical Physics Unit, University Hospital Hassan II, Morocco
}

*Corresponding author: Abboud Fatima Zahra, Hospital Center University Hassan II, Fes, Morocco, Tel: +212627782466; E-mail: abboud.fatimazahra@gmail.com

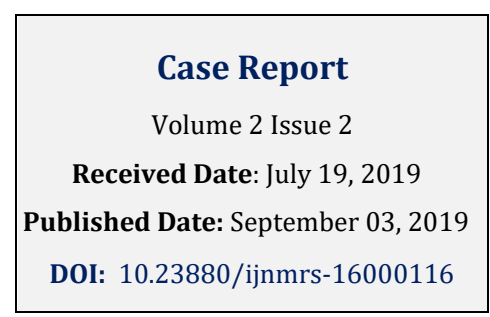

Received Date: July 19, 2019

DOI: $10.23880 /$ ijnmrs-16000116

\section{Abstract}

Background: We report here a case of nasal cavity chondrosarcoma and its treatment.

Methods: A women aged of 29 years old presented a long history of nasal obstruction. He was diagnosed with nasal cavity chondrosarcoma. Loco regional extension in the CT scan showed involvement of the right nasal cavity, and suspected involvement of the medial wall of the orbit. She had surgical excision with removal of the tumor. Given the positive margins, adjuvant radiotherapy was indicated. The radiotherapy was delivered with intensity modulated radiation therapy (IMRT) technique in order to overcome anatomical complexity of the region, cover the tumor bed and preserve the organs at risk.

Results: The patient remains without recurrence with more than 12 months of follow-up.

Conclusions: Radical excision of the tumor is the treatment of choice, but our results show a high level of efficacy for fractionated photon radiotherapy after surgery, in keeping with other series, particularly now with the advent of new radiotherapy techniques. This should encourage the further development of sophisticated photon radiotherapy.

Keywords: Chondrosarcoma; Nasal Cavity; Radiotherapy; IMRT; Cellularity

Abbreviations: IMRT: Intensity Modulated Radiation Therapy; CHS: Chondrosarcoma; CT: Computed Tomographic; MR: Magnetic Resonance; SIB: Simultaneous Integrated Boost; MRI: Magnetic Resonance Imaging; EEAs: Endoscopic Endonasal Approaches.

\section{Introduction}

Chondrosarcoma (CHS) is a rare disease that accounts for approximately $0.15 \%$ of all intracranial tumors and $6 \%$ of skull base tumors [1]. The 2013 WHO classification effected a significant change in the grading and classification for chondrosarcoma, which now separates grade 1 chondrosarcoma from grade 2 and grade 3 tumors. The most important prognostic predictor for recurrence and metastasis of chondrosarcoma is the histologic grade, which is assigned on a 3-tier system based on nuclear size, hyperchromasia, cellularity, and mitotic count; some tumors show coexistence of various histologic grades and the highest grade present should be reported [2]. It arise anywhere along the length of the spine, but again frequently occur at the skull base where 
they arise from cartilaginous elements (mesenchymal cells) near fused joints and have a predilection for spheno-petrosal and spheno-occipital synchondrosis, as well as the nasal cavity and paranasal sinuses [3]. On computed tomographic (CT) and magnetic resonance (MR) examinations, they have well-described imaging features that help to distinguish them from each other and from other skull base lesions. Imaging also defines the tumor's relationship to adjacent nerves, vessels, and bones, allowing for detailed surgical and radiation treatment planning. After treatment, imaging helps to evaluate for treatment-related complications and tumor recurrence.

The growth rates of these tumours are characteristically slow. Nevertheless, they are locally invasive and have a high tendency for local recurrence. Evans HL, et al. [4] reported metastasis rates of chondrosarcomas of $0 \%$ of grade $1,10 \%$ of grade 2 and $71 \%$ of grade 3 tumours. Pathological specimens are essential for definitive diagnosis. Chondrosarcoma may contain myxoid stroma, and for less typical tumours the histological differentiation between chordoma and chondrosarcoma may be difficult. The rarity of these tumours makes diagnosis and management difficult, and a multidisciplinary approach is paramount. Surgery is a key component in the successful management of these patients [5-7], and is typically followed by radiotherapy. Although not formally tested in randomised trials, there is a reasonable evidence base for this approach [5,6,8-11]. Because these tumours are considered to be relatively radiation resistant, high doses are needed for successful control $[3,10]$. Between 60 and $65 \mathrm{~Gy}$ is considered a minimum useful dose, but higher doses are favoured, with higher doses normally being delivered with charged particle irradiation. The sino-nasal region is difficult to treat and delivering radical doses of radiotherapy is challenging. IMRT radiation technique helps treating this site with a good compromise between efficacy and safety.

\section{Case Report}

We report here the case of a 29 -year-old patient with a history of unilateral right nasal obstruction of progressive worsening for one year before her consultation. The patient also reports a decrease in visual acuity in the right eye for a few months, becoming right blindness 2 months before admission. She denied any nasal bleeding, loss, fevers, chills or fatigue. There was no history of smoking or intake of alcohol. Physical examination demonstrated a well-developed and well-nourished woman in no distress. Intranasal examination by anterior rhinoscopy revealed a high-grade intranasal obstruction, chiefly right-sided, and a purulent right nasal discharge. The nasal septum was crowded bilaterally, making instrumentation in the nostrils difficult. At the ophthalmological examination the patient was in right blindness, with a luminous perception and a photo-motor reflex abolished. When examining the lymph node areas there was no lymphadenopathy. Laboratory evaluations including complete blood count, electrolytes and basic metabolic profile were normal. The patient was investigated with a computerized tomography (CT) scan of the cervicofacial floor confirmed a hypodense tissue mass in the nasal cavity, with irregular contours, measuring $51 \mathrm{~mm} * 32 \mathrm{~mm} * 55 \mathrm{~mm}$ (Figures $1 \mathrm{~A} \&$ B), this mass blows the bone of the body of the sphenoid with rupture of the cortex in places. It has an endocerebral extension with infiltration of the right optic nerve at the top (Figures 2A \& B) and an invasion of the hard palate at the bottom (Figures 3A \& B). The patient also had an MRI (Figure 4) that confirmed the invasion of the right optic nerve, sphenoidal bone and hard palate. Further, staging assessment, which included CT scan of the chest and abdomen, was carried out with no evidence of distant metastasis. The tumor has been classified T4N0M0 according to the 8th edition of the AJCC/IUCC. The patient underwent a biopsy was taken under general anesthesia of the tumor by endoscopy of the right nasal cavity, which was suggestive of chondrosarcoma.

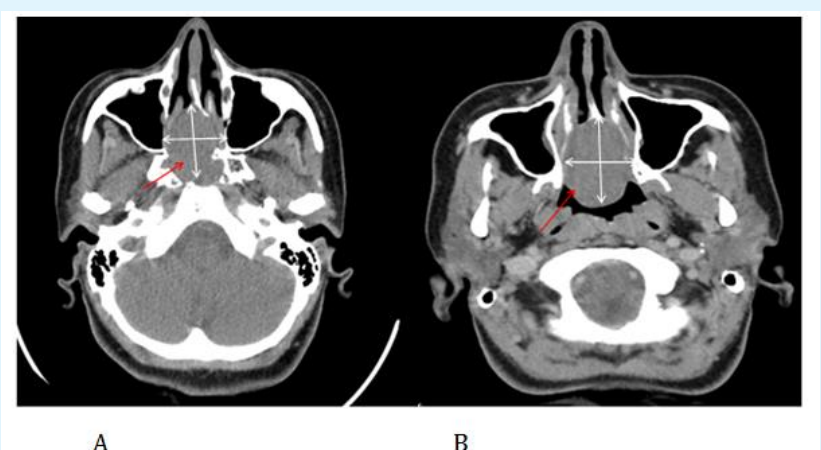

Figures 1A \& B: Axial CT scan slide showing the cavity nasal tumor. 


\section{International Journal of Nuclear Medicine \& Radioactive Substances}

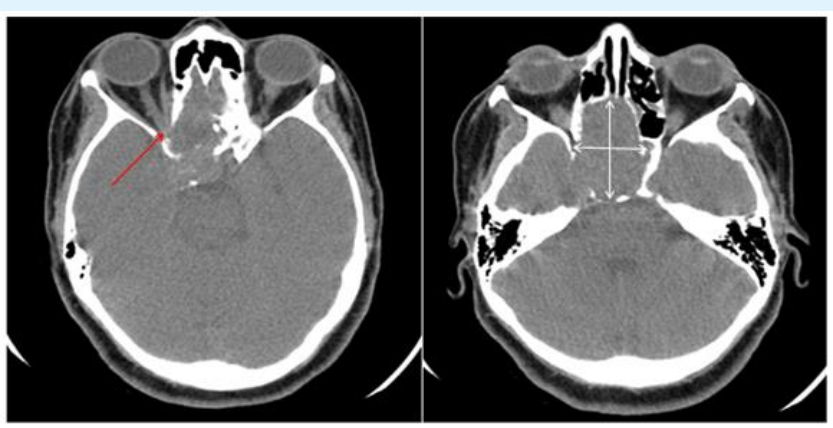

A

B

Figures 2A \& B: Axial scan sections showing invasion of the sphenoidal bone and optic nerve.

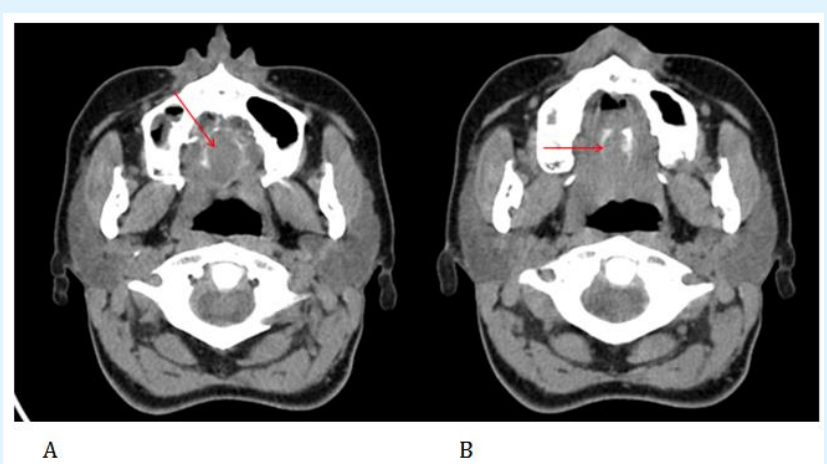

Figures 3A \& B: Axial scan sections showing invasion of the hard palate.
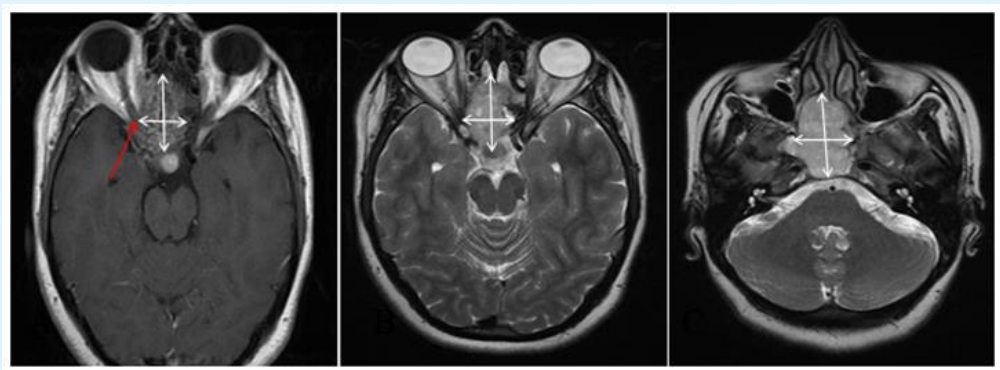

A

B

C
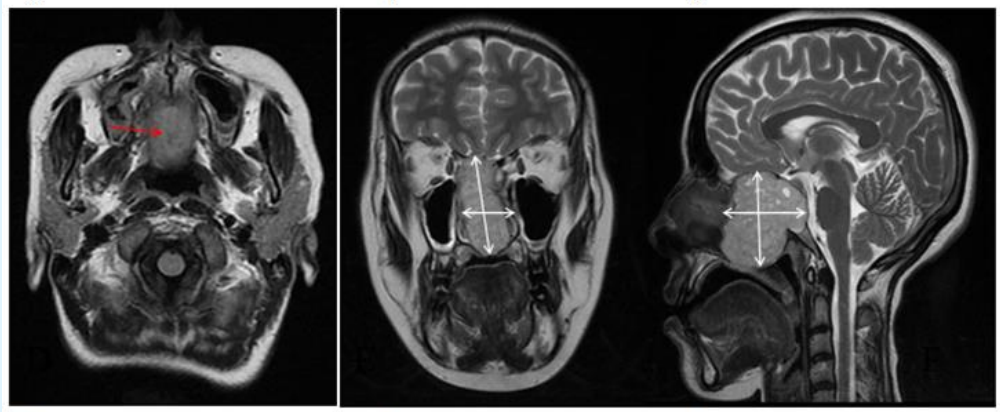

E

Figure 4: MRI sections in the axial plane sequence T1 (A), sequence T2 (B,C,D), frontal plane (E) and sagittal plane (F) showing invasion of the optic nerve, sphenoidal bone body and hard palate. 
The patient underwent a tumor excision with a right lateral endoscopic endonasal approach. The surgery removed the maximum tumor bulk from the nasal cavity. The histopathology of the surgical specimen was consistent with grade II chondrosarcoma. The margins of surgical excision were positive. This case was then discussed in a head and neck tumor board. Considering the positivity of the surgical margins as well as the extent of the tumor, it was staged as T4N0M0 as per the 2017 AJCC Staging (American Joint Committee on Cancer staging system). Adjuvant radiotherapy was decided. He was planned for a total dose of 66 Gy of RT in 33 fractions, at a daily dose fraction of 2 Gy over 6.5 weeks using the intensity-modulated radiotherapy (IMRT) technique with fields prescribed to the $100 \%$ isodose line in simultaneous integrated boost (SIB), using 6-MV photons. We were able to keep the tolerance doses of organs at risk such as the optic chiasma, optic nerves, retina, pituitary and parotids within normal limits and at the same time deliver the intended dose of radiation to the tumor site (Figures $5 \& 6$ ). The patient received treatment according to plan and the tolerance was good. She was reviewed weekly during the treatment by our team. There were no significant side effects observed except moderate skin reactions on the irradiated skin and occasional right nasal blockage at the end of treatment.

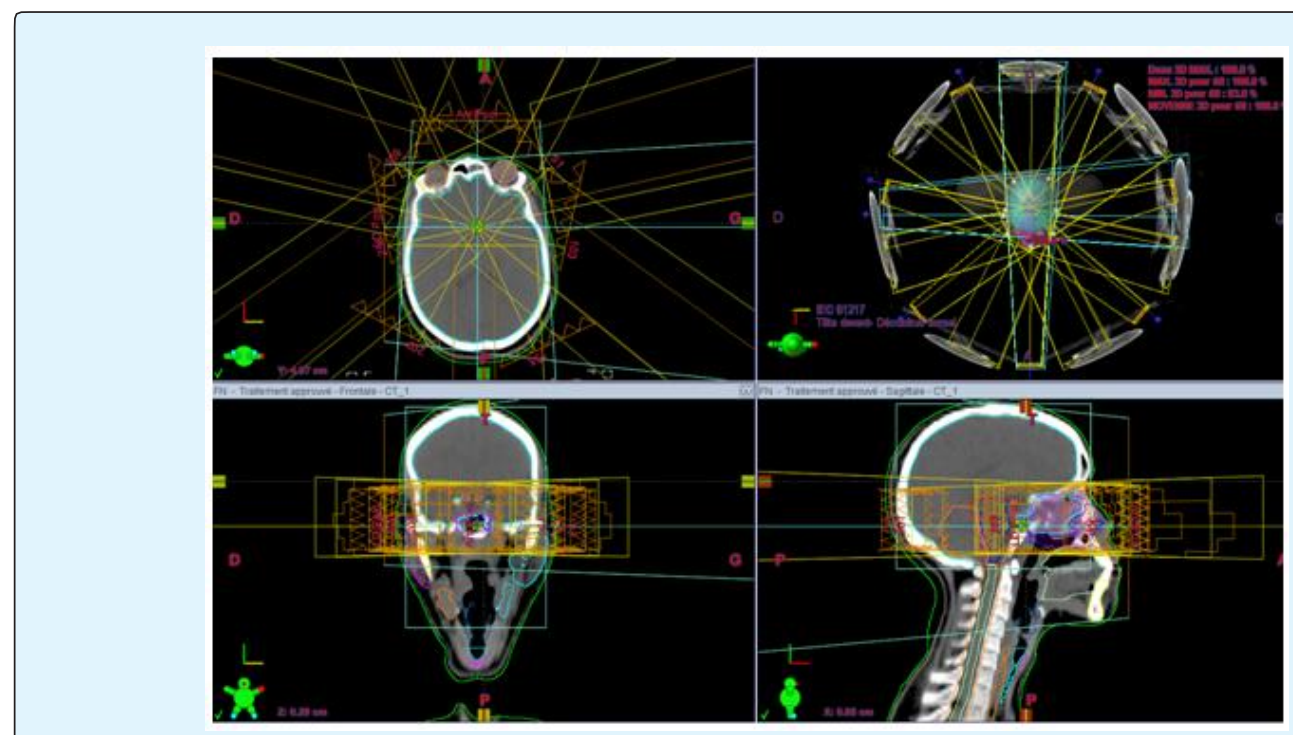

Figure 5: Disposition of multiple IMRT beams on the three plans (axial, sagittal and coronal).

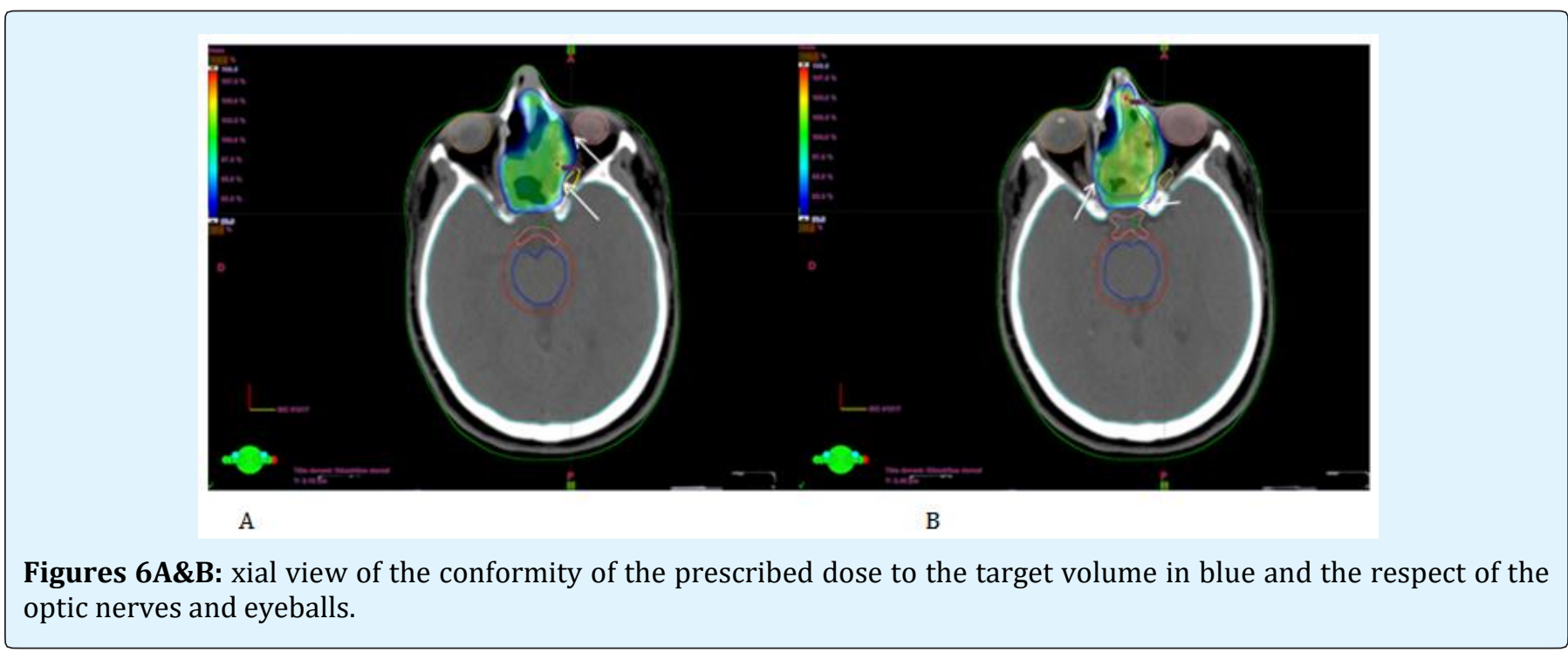




\section{Results}

The patient was reviewed after 1 month. Skin reactions almost resolved. A CT scan performed 2 months after the end of treatment that showed a complete response to the tumor (Figure 7). The patient did not present a local-regional recurrence or metastasis for more than 12 months of follow-up.

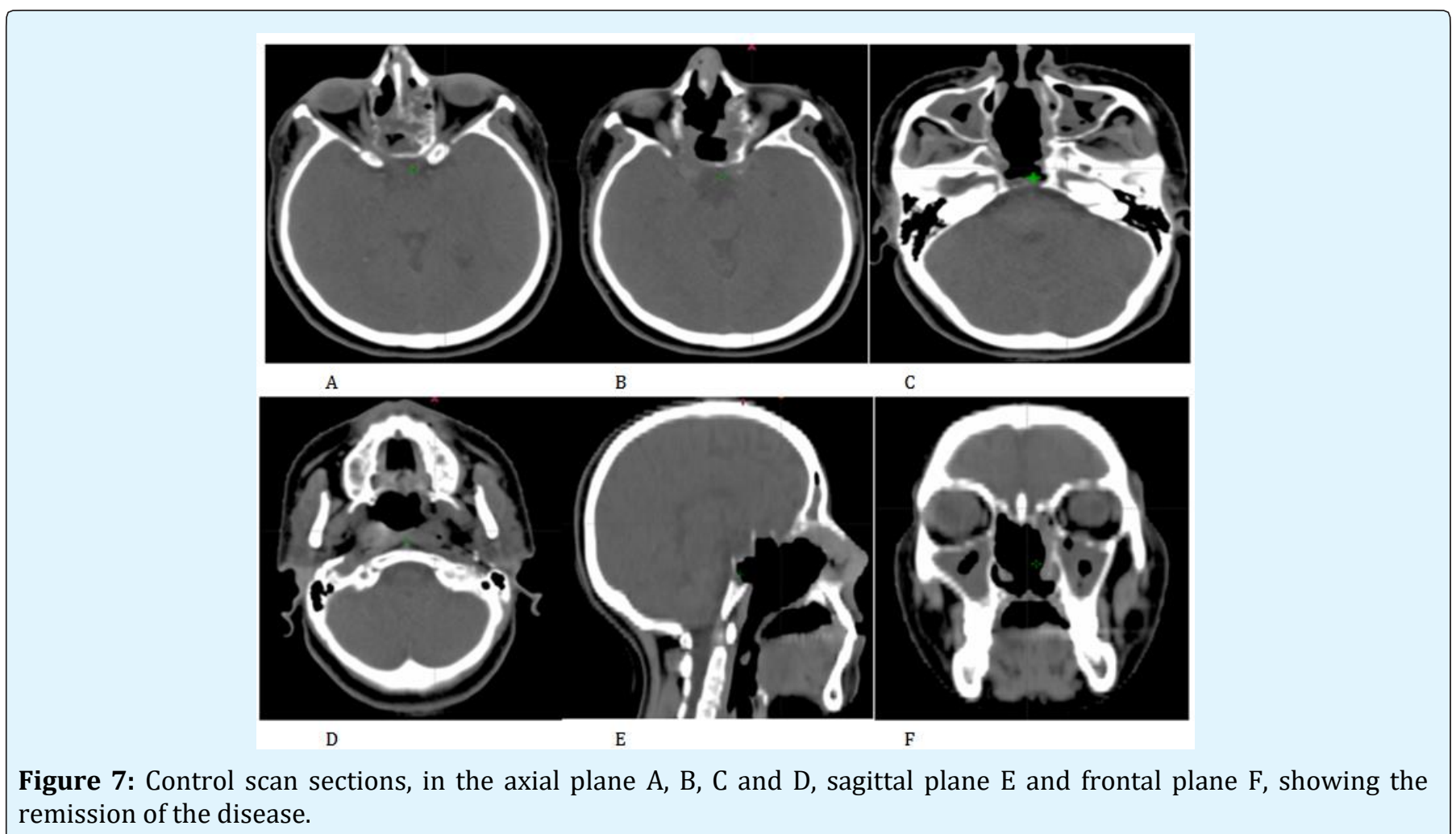

\section{Discussion}

Chondrosarcoma (CHS) is an uncommon intranasal tumor. In any location it is preeminently a neoplasm found in adults 25 to 50 years of age. It is a slow growing malignant tumour with hyaline cartilaginous differentiation, accounting for approximately $20 \%$ of allmalignant bone tumours. Primary chondrosarcomas have been further categorized by the histologic subtype and can be defined as conventional intramedullary, clear cell, myxoid, mesenchymal, or dedifferentiated variants. The conventional intramedullary chondrosarcoma sub type accounts for the majority (about 85\%-90\%) of skull base chondrosarcomas and has the best-described imaging characteristics [12]. One review of the literature revealed no case reports of clear cell or differentiated subtypes occurring at the skull base [12]. The myxoid subtype is most commonly encountered in an extraskeletal location and fewer than 15 cases have been reported at the skull base [13]. Mesenchymal sub types account for most of the remaining skull base chondrosarcomas and are associated with higher mortality and poorer prognosis [12]. Chondrosarcomas are generally less aggressive than other types of sarcomas in the head and neck and usually behave only as locally destructive tumours. Lymphatic invasion or lymphnode deposits are unusual. Metastases occurring late, via blood vessels, with spread to distant organs, particularly lung and bones [14].

Although chondrosarcomas of the head and neck generally show a peak incidence in the fourth decade, a case has been reported in a sixteen month- old infant [15]. Myers Em, et al. [16] further suggest a higher incidence in men than women in the head and neck region (M:F ratio 10:1). Nishizawa $S$, et al. [17], however, reported an almost equal sex distribution for chondrosarcomas of the nasal septum. Signs and symptoms depend upon the anatomical location of the turnout and its relationship to critical structures, both intra- and extra-cranially. Early 
symptoms are rather vague and long delays in proper diagnosis are frequently reported. Tumours may show an extensive involvement of the base of the skull, and in such a case palsies of the $2^{\text {nd }}, 3^{\text {rd }}, 4^{\text {th }}, 5^{\text {th }}$ and $6^{\text {th }}$ cranial nerves may be present. When the tumour involves the foramina and enters the cranium, symptomatology from the $9^{\text {th }}$, $10^{\text {th }}, 11^{\text {th }}$ and $12^{\text {th }}$ cranial nerves may occur. Further symptoms may be present either from the expansion of the lesion anteriorly to the sphenoid sinus and the ethmoids or they can result from increased intracranial pressure [18]. The interval between onset of symptoms and final diagnosis may vary and in some series is reported to be between 18 and 60 months [18]. Patients with chondrosarcomas may also present with nasopharyngeal symptoms. Nasal and eustachian tube obstructions, throat fullness, epistaxis with the presence of a nasal mass, paralysis of the tongue, dysphagia and dysarthria are some of the symptoms encountered $[19,20]$. Pain or hypaesthesia in the distribution of the trigeminal nerve alone or in association with symptoms resulting from facial and acoustic nerve involvement can also be the initial manifestations. Visual loss, as in our case, as the presenting symptom is rather uncommon and usually occurs in lesions with expansion to the anterior part of the skull base. It is interesting to note that although visual loss as a result of chondrosarcomas is rather common, decreased visual acuity in patients with good vision is infrequently encountered.

Blindness seems to occur very rapidly when the tumour invades the optic chiasma and the optic nerve without a transient period of a gradual decreasing visual acuity. Rapid visual loss can be misleading and may be attributed to optic neuritis, the reason being that in chondrosarcomas fundoscopic findings are usually absent [21]. Commonly reported symptoms include diplopia (51\%), headache (31\%), decreased hearing, dizziness, and vestibular disturbance (21\%). Chondrosarcomas of the skull base have also been associated with the clinical course of a variety of heterogenous conditions including Paget's disease, osteocartilaginous exostoses, Maffucci syndrome, Ollier's disease and osteochondromas, but the association of these conditions with the presence of chondrosarcomas has not been documented [22]. Clinically, as in our case, chondrosarcomas of the nasal cavity are painless, slowly-growing tumours. Nasal obstruction, facial swelling, nasal discharge, proptosis and facial numbness are the most common presenting features. It is not uncommon for patients suffering from chondrosarcomas of the base of the skull to be sent for radiological examinations several months or even years following treatment for headaches and other vague neurological symptoms, having been misdiagnosed as suffering from disorders of psychosomatic origin.

Plain radiographs have little to offer. Computerized Tomography (CT) and Magnetic Resonance Imaging (MRI) are usually sufficient to assess the extent of the disease. To determine the relationship of these tumours to vascular structures, especially in cases with temporal extension, Magnetic Resonance Angiography (MRA), and digital as well as conventional angiography, is used [2224]. The bony destruction of the skull base by chondrosarcomas is best seen using CT, which has the advantage of showing areas of bone destruction and the ability to delineate areas of calcification and mineralization within the tumour [22]. Most of the tumours show marked contrast enhancement of their soft tissue components [25]. The demonstration of tumour margins in relation to the brain and other extracranial soft tissues cannot always be defined. In those cases, MRI is beneficial, especially when combined with a fat suppression technique [26].

MRI is recommended not only for the evaluation of soft tissue changes but also for evaluating the lesions post-operatively [27]. It can also provide information on the anatomical location of the tumour in relation to neighbouring neurovascular structures, thus diminishing the necessity for further angiographic studies. On MRI, chondrosarcomas often have low to intermediate signal intensity on T1-weighted images and very high signal intensity on T2-weighted images. Chondrosarcomas do not generally show restricted diffusion, and they have higher apparent diffusion coefficient values than chordomas [28]. Skull base chondrosarcomas can have either heterogeneous or homogeneous enhancement patterns. The solid component of the tumor typically demonstrates intense homogeneous enhancement after contrast administration, but there may also be a pattern of peripheral enhancement with internal lines and swirls of enhancement [28]. The presence of calcifications alters the internal signal characteristics of chondrosarcoma, and mineralized lesions often have lower signal intensity on T2-weighted images and more commonly show heterogeneous gadolinium enhancement [28].

In terms of therapy, recommended treatment for these lesions is variable. Myers Em, et al. [16] suggests that the treatment of choice is radical excision whereas Fu YS, et al. [29] prefer adequate local excision, in the form of a block resection. Radiotherapy is not a first-line treatment and is best reserved for recurrences [30]. Nishizawa $S$, et al. [17] is more aggressive using radical excision with preand post-operative radiotherapy and chemotherapy in 
their treatment regime. Surgically, taking into account the overall biological behaviour of head and neck CHS, the most effective treatment modality for this neoplasia is aggressive surgery with wide en-bloc resection, ensuring that an adequate histologically clear margin is obtained, since residual disease is known to be an important cause of recurrence [31-33], but the potential advantages of endoscopic endonasal approaches (EEAs) include the possibility of accessing multiple skull base compartments, even bilaterally, in a single procedure while avoiding retraction or manipulation of neurovascular structures; this is exceptionally displayed during endonasal resection of chondrosarcomas [34-37]. Endonasal endoscopic resection of petroclival chondrosarcomas appears to be a safe and feasible technique, capable of achieving total or near total removal in most cases, despite involvement of surrounding regions, with low complication rates. Nonetheless, further studies with a greater number of patients are necessary to confirm these initial impressions [38].

Although surgery is a key component in the successful management of these patients [6-8,39]. Radiotherapy is of paramount importance in the management chondrosarcomas, if sufficient dose can be given. The existence of a dose response is now well established $[3,10,11,40,41]$, and clear evidence is emerging that the size of the tumour affects the ultimate outcome. This may apply to the preoperative volume [7] or the size of the residuum after surgery [10]. A careful assessment of this factor might contribute to the surgical management of cases, as well as guiding the radiotherapy. Thus radiotherapy is indicated in instances in which tumours are considered incompletely resected or unresectable $[32,33,42,43]$. The use of adjuvant treatment modalities in high grade tumours has been suggested10, but their real efficacy in these cases remains to be fully investigated.

Potluri S, et al. [44] showed in their series consisting of the nineteen patients referred to the Neuro-Oncology Unit at Addenbrooke's Hospital (Cambridge, UK) between 1996 and 2009 and treated with photon radiotherapy were reviewed, encouraging results in proving the efficacy of sophisticated high dose photon radiotherapy, after tumour resection by a specialist surgical team. This is particularly important for those patients with contraindications to hadron therapy. The results also suggest that a small volume of residual tumour may be controlled, even with photon treatment. This might allow less morbid surgery, within the context of a multidisciplinary management approach. Modern radiotherapy techniques such as IMRT allow high doses to target volumes, while sparing organs at risk such as the optic chiasma, optic nerves, retina, pituitary, parotids, temporal lobes and auditory structures (including the cochlea and optic structures)[45]. Chemotherapy does not appear to have a significant effect on survival $[32,46]$ and it is generally accepted that this treatment modality should be used for palliative purpose, in association or not with surgery, and radiotherapy in cases of disseminated diseases. According to the clinical series reviewed, the 5-year survival rate for head and neck $\mathrm{CSH}$ varies between $32 \%$ and $87.5 \%[47,48]$. This variation may be explained by the scant and heterogeneous clinical series that usually include different histological variants of CHS in the same analysis and the advancements in surgery that directly improved the survival rates.

\section{Conclusion}

Chondroma and chondrosarcoma rarely involve paranasal sinuses and intranasal structures. The intricate anatomy involved usually precludes complete eradication in the sense of eradication with safe margin. The results of irradiation therapy are encouraging. Rhinologists confronted with this problem must decide whether circumstances justify conservative treatment, with probable recurrences, or whether they demand radical procedures planned to destroy the tumor completely, thereby risking undesirable functional and cosmetic results.

\section{References}

1. Cianfriglia F, Pompili A, Occhipinti E (1978) Intracranial malignant cartilaginous tumours. Report of two cases and review of literature. Acta Neurochir (Wien) 45(1-2): 163-175.

2. Fletcher CDM, Bridge JA, Hogendoorn P, Mertens, F (2013) Pathology and genetics of tumours of soft tissue and bone. WHO classification of tumours, $3^{\text {rd }}$ (Edn.), Vol 5, IARC Publications.

3. Schulz-Ertner D, Karger CP, Feuerhake A, Nikoghosyan A, Combs SE, et al. (2007) Effectiveness of carbon ion radiotherapy in the treatment of skullbase chordomas. Int J Radiat Oncol Biol Phys 68(2): 449-457.

4. Evans HL, Ayala AG, Romsddahl MM (1977) Prognostic factors in chondrosarcomsa of bone: a clinicopathologic analysis with emphasis on histologic grading. Cancer 40(2): 818-831. 


\section{International Journal of Nuclear Medicine \& Radioactive Substances}

5. Tai PT, Craighead P, Liem SK, Jo BH, Stitt L, et al. (2000) Management issues in chordoma: a case series. Clin Oncol (R Coll Radiol) 12(2): 80-86.

6. Crockard HA, Steel T, Plowman N, Singh A, Crossman J, et al. (2001) A multidisciplinary team approach to skull base chordomas. J Neurosurg 95 (2): 175-183.

7. Jawad MU, Scully SP (2010) Surgery significantly improves survival in patients with chordoma. Spine 35(1): 117-123.

8. Tai PT, Craighead P, Bagdon F (1995) Optimization of radiotherapy for patients with cranial chordoma. A review of dose-response ratios for photon techniques. Cancer 75(3): 749-756.

9. Foweraker KL, Burton KE, Maynard SE, Jena R, Jefferies SJ, et al. (2007) High-dose radiotherapy in the management of chordoma and chondrosarcoma of the skull base and cervical spine: Part 1--clinical outcomes. Clin Oncol 19(7): 509-516.

10. Ares C, Hug EB, Lomax AJ, Bolsi A, Timmermann B, et al. (2009) Effectiveness and safety of spot scanning proton radiation therapy for chordomas and chondrosarcomas of the skull base: first long-term report. Int J Radiat Oncol Biol Phys 75(4): 1111-1118.

11. DeLaney TF, Liebsch NJ, Pedlow FX, Adams J, Dean S, et al. (2009) Phase II study of high-dose photon/proton radiotherapy in the management of spine sarcomas. Int J Radiat Oncol Biol Phys 74(3): 732-739.

12. Bloch OG, Jian BJ, Yang I, Han SJ, Aranda D, et al. (2009) A systematic review of intracranial chordosarcoma and survival. J Clin Neurosci 16(12): 1547-1551.

13. Arpino L, Capuano C, Gravina M, Franco A (2011) Parasellar myxoid chondrosarcoma: a rare variant of cranial chondrosarcma. J Neurosurg Sci 55(4): 387389.

14. Batsakis JG, Solomon AR, Rice DH (1980) The pathology of head and neck tumours: Neoplasms of cartilage, bone and the notochord, part 7. Head and Neck Surgery 3(1): 43-57.

15. Gallagher TM, Strome M (1972) Chondrosarcoma of the facial region. Laryngoscope 82(6): 978-984.

16. Myers EM, Thawley SE (1979) Maxillary chondrosarcoma. Arch Otolaryngol 105: 116-118.
17. Nishizawa S, Fukaya T, Inouge K (1984) Chondrosarcoma of the nasal septum: a report of an uncommon lesion. Laryngoscope 94(4): 550-553.

18. Volpe NJ, Liebsch NJ, Munzenrider JE, Lessell S (1993) Neuro-ophthalmologic findings in chordoma and chondrosarcoma of the skull base. Am J Ophthalmol 115(1): 97-104.

19. Batsakis JG, Kittleson AC (1963) Chordomas. Otolaryngologic presentation and diagnosis. Arch Otolaryngol 78: 168-175.

20. Perzin KH, Pushparaj N (1986) Nonepithelial tumors of the nasal cavity, paranasal sinuses, and nasopharynx, A clinicopathological study, XIV: Chordomas. Cancer 57(4): 784-796.

21. Bagan SM, Hollenhorst RW (1980) Ocular manifestations of intracranial chordomas. Trans Am Ophthalmol Soc 78: 148-155.

22. Weber AL, Brown EW, Hug EB, Liebsch NJ (1995) Cartilaginous tumors and chordomas of the cranial base. Otolaryngol Clin North Am 28 (3): 453-471.

23. Yaghmai I (1978) Angiographic features of chondromas and chondrosarcomas. Skeletal RadioI 3(2): 91-98.

24. Meyers SP, Hirsch WL, Curtin HD, Sekhar LN, Sen C, et al. (1992) Chondrosarcomas of the skull base: MR Imaging features. Radiology 184(1): 103-108.

25. Oot RF, Melville GE, New PF, Austin-Seymour M, Munzenrider J, et al. (1988) The role of MR and CT in evaluating clival chordomas and chondrosarcomas. Am J Roentgenol 151(3): 567-575.

26. Tien RD (1992) Fat-suppression MR imaging in neuroradiology: techniques and clinical application. Am J Roentgenol 158(2): 369-379.

27. Brown E, Hug E, Weber AL (1994) Chondrosarcoma of the skull base. Neuroimaging Clinics of North America 4: 529-541.

28. Golden L, Pendharkar A, Fischbein N (2018) Chapter 7-Imaging Cranial Base Chordoma and Chondrosarcoma., Chordomas and Chondrosarcomas of the Skull Base and Spine 2nd (Edn.), pp: 67-78.

29. Fu YS, Perzin KH (1974) Non-epithelial tumours of the nasal cavity, paranasal sinuses and nasopharynx: A clinicopathologic study, 3, Cartilaginous tumors 


\section{International Journal of Nuclear Medicine \& Radioactive Substances}

(chondroma, chondrosarcoma). Cancer 34(2): 453463.

30. Finn DG, Geopfert H, Batsakis JG (1984) Chondrosarcoma of the head and neck. Laryngoscope 94(12): 1539-1544.

31. Garzino-Demo P, Tanteri G, Boffano P, Ramieri G, Pacchioni D, et al. (2010) Chondrosarcoma of the temporomandibular joint: a case report and review of the literature. J Oral Maxillofac Surg 68(8): 20052011.

32. Ormiston IW, Piette E, Tideman H, Wu PC (1994) Chondrosarcoma of the mandible presenting as periodontal lesions: report of 2 cases. J Craniomaxillofac Surg 22(4): 231-235.

33. Prado FO, Nishimoto IN, Perez DEC, Kowalski LP, Lopes MA (2009) Head and neck chondrosarcoma: analysis of 16 cases. Br J Oral Maxillofac Surg 47(7): 555-557.

34. Mesquita Filho PM, Ditzel Filho LF, Prevedello DM, Martinez CA, Fiore ME, et al. (2014) Endoscopic endonasal surgical management of chondrosarcomas with cerebellopontine angle extension. Neurosurg Focus 37(4): E13.

35. Frank G, Sciarretta V, Calbucci F, Farneti G, Mazzatenta D, et al. (2006) The endoscopic transnasal transsphenoidal approach for the treatment of cranial base chordomas and chondrosarcomas. Neurosurgery 59(1): 50-57.

36. Moussazadeh N, Kulwin C, Anand VK, Ting JY, Gamss $C$, et al. (2015) Endoscopic endonasal resection of skull base chondrosarcomas: technique and early results. J Neurosurg 122(4): 735-742.

37. Zhang Q, Kong F, Yan B, Ni Z, Liu H (2008) Endoscopic endonasal surgery for clival chordoma and chondrosarcoma. ORL J Otorhinolaryngol Relat Spec 70(2): 124-129.

38. Ditzel Filho LF, Prevedello DM, Dolci RL, Jamshidi AO, Kerr EE, et al. (2015) The Endoscopic Endonasal Approach for Removal of Petroclival Chondrosarcomas. Neurosurg Clin N Am 26(3): 453462.
39. Crockard HA, Cheeseman A, Steel T, Revesz T, Holton $\mathrm{JL}$, et al. (2001) A multidisciplinary team approach to skull base chondrosarcomas. J Neurosurg 95(2): 184189.

40. Mendenhall WM, Mendenhall CM, Lewis SB, Villaret DB, Mendenhall NP (2005) Skull-base chordoma. Head Neck 27(2): 159-165.

41. Romero J, Cardenes H, la Torre A, Valcarcel F, Magallon R, et al. (1993) Chordoma: results of radiation therapy in eighteen patients. Radiother Oncol 29(1): 27-32.

42. Gadwal SR, Fanburg-Smith JC, Gannon FH, Thompson LDR (2000) Primary chondrosarcoma of the head and neck in pediatric patients: a clinicopathologic study of 14 cases with a review of the literature. Cancer 88(9): 2181-2188.

43. Kainuma K, Netsu K, Asamura K, Hayashi K, Takumi Y, et al. (2009) Chondrosarcoma of the nasal septum: a case report. Auris Nasus Larynx 36(5): 601-605.

44. Potluri S, Jefferies SJ, Jena R, Harris F, Burton KE, et al. (2011) Residual postoperative Tumour Volume Predicts Outcome after High-dose Radiotherapy for Chordoma and Chondrosarcoma of the Skull Base and Spine. Clin Oncol 23(3): 199-208.

45. Lohia S, Rajapurkar M, Nguyen SA, Sharma AK, Gillespie MB, et al. (2014) A comparison of outcomes using intensity-modulated radiation therapy and 3dimensional conformal radiation therapy in treatment of oropharyngeal cancer. JAMA Otolaryngol Head Neck Surg 140(4): 331-337.

46. Gorsky M, Epstein JB (2000) Craniofacial osseous and chondromatous sarcomas in British Columbia: a review of 34 cases. Oral Oncol 36(1): 27-31.

47. Lee SY, Lim YC, Song MH, Seok JY, Lee WS, et al. (2005) Chondrosarcoma of the head and neck. Yonsei Med J 46(2): 228-232.

48. Garrington GE, Collett WK (1988) Chondrosarcoma, II, Chondrosarcoma of the jaws: analysis of 37 cases. J Oral Pathol \& Med 17(1): 12-20. 